\title{
Introduction to the Special Issue on Terahertz Cameras and Detector Arrays
}

\author{
Sascha Preu ${ }^{1}$ (D)
}

Received: 23 July 2015 / Accepted: 26 July 2015/

Published online: 18 August 2015

(C) Springer Science+Business Media New York 2015

\begin{abstract}
Already an old saying states that "a picture is worth a thousand words". Imaging allows to obtain complex information in a very compressed, but still comprehensible way-not only in the visible. Terahertz $(\mathrm{THz})$ radiation $(0.1-10 \mathrm{THz})$ features a combination of unique properties, particularly relevant for imaging applications, including penetration through many optically opaque media, (much) higher spatial resolution than in the microwave range due to the shorter wavelength, harmlessness at the available power levels, and even the possibility of "spectroscopic imaging" by fingerprinting of a manifold of resonances that many substances feature in the Terahertz range. Therefore, $\mathrm{THz}$ imaging is highly attractive to key applications such as in homeland security, where scanning for suspicious objects hidden in envelopes or beneath clothing allows for threat detection, non-destructive testing of industrial products for quality control, and applications in astronomy where $\mathrm{THz}$ radiation can penetrate through stellar dust and nebulas, being able to identify their chemical constituents while visible light or X-rays are severely scattered. Therefore, huge efforts have been undertaken in the past years to develop high sensitivity $\mathrm{THz}$ imaging arrays, cameras, and systems; with great progress, yet reaching real-time operation with sufficient dynamic range for many applications. In contrast to the visible, where one technology dominates the market, several approaches are currently followed up in the $\mathrm{THz}$ range, tailored to specific target applications. This special issue covers seven invited articles from distinguished groups all around the globe, reviewing the state-of-the art in $\mathrm{THz}$ camera development and showing latest results on and technical details of various approaches:
\end{abstract}

1. The paper Passive $350 \mathrm{GHz}$ Video Imaging Systems for Security Applications by E. Heinz, T. May, D. Born, G. Ziegler, S. Anders, V. Zakosarenko,

S. Preu

preu@imp.tu-darmstadt.de

1 Terahertz Systems Technology, Department of Electrical Engineering and Information

Technology, Technische Universität Darmstadt, Merckstr. 25, 64283 Darmstadt, Germany 
H.-G. Meyer, and C. Schäffel, (a collaboration between Supracon AG, Jena, Germany, the Leibnitz Institute of Photonic Technology, Jena, Germany, and the Institut für Mikroelektronik und Mechatronik Systeme, Ilmenau, Germany) presents latest results on a passive, background-limited, real-time $\mathrm{THz}$ linear receiver array using superconducting transition edge sensors, operated at temperatures below $1 \mathrm{~K}$. The camera can detect a temperature difference well below 1 $\mathrm{K}$ within a frequency window of $350 \pm 40 \mathrm{GHz}$. A camera prototype for stand-off imaging and first images is presented.

2. The article Terahertz heterodyne array receivers for astronomy by U. Graf, C.E. Honingh, K. Jacobs and J. Stutzki (Univeristy of Cologne, Germany) reviews the history and the state-of-the art in superconducting $\mathrm{THz}$ heterodyne receivers. The authors also discuss suitable choices for local oscillators, and optics, the cryostate design and IF back ends.

3. The paper Review of terahertz technology development at INO by D. Dufour et al. from the Institut National d'Optique (INO), Canada, describes the technical components of the IRXCAM-THz series, their uncooled VOx microbolometerbased cameras. Further, several imaging applications are presented.

4. An uncooled VOx Microbolometer Terahertz Focal Plane Array and Camera with Improved Sensitivity in Sub-Terahertz Region is detailed by N. Oda, S. Kurashina, M. Miyoshi, K. Doi, T. Ishi, T. Sudou, T. Morimoto, H. Goto and T. Sasaki, NEC Corporation, Japan. Each pixel contains a THz cavity and a read out integrated circuit. Minimum detectable power levels have been determined at various $\mathrm{THz}$ frequencies and are compared to other camera concepts.

5. Terahertz real-time imaging uncooled arrays based on antenna-coupled bolometers or FET developed at CEA-Leti is contributed by F. Simoens, J. Meilhan, and J.-A. Nicolas from the CEA Leti-MINATEC, France. It discusses bolometer focal plane arrays using pixels with a resonant $\mathrm{THz}$ cavity. Further, antenna designs and chip layouts for dual polarization imaging are presented. The second part of the paper shows first results on field effect transistor (FET) rectifier focal plane arrays based on CMOS technology with integrated read out circuitry.

6. A room-temperature operated Camera for High-Speed $\mathrm{THz}$ Imaging, consisting of an NMOS field effect transistor array with two rectifying transistors per pixel is introduced by J. Zdanevičius, M. Bauer, S. Boppel, V. Palenskis, A. Lisauskas, V. Krozer, and H. Roskos, (Vilnius University, Lithuania, and Goethe University, Frankfurt, Germany). Emphasis is put on noise reduction in the read out electronics.

7. Further camera concepts with silicon-based MOSFETs or SiGe heterojunction bipolar transistors (HBTs) are presented in the contribution $\mathrm{THz}$ direct detector and heterodyne receiver arrays in silicon nanoscale technologies by J. Grzyb and U. Pfeiffer, University of Wuppertal, Germany. The manuscript particularly focuses on design considerations and integration aspects. Finally, results on heterodyne, multicolor receiver arrays are presented.

Acknowledgments The guest editor would like to thank all contributors and reviewers for their valuable contributions and hopes that the readers enjoy this special issue. 